\title{
Médiévales
}

Langues, Textes, Histoire

65 | automne 2013

Le couple dans le monde franc

\section{Le couple en droit au haut Moyen Âge : autour de l'affectio maritalis et des relations patrimoniales}

The Couple in Front of the Law in the Early Middle Ages: Affectio Maritalis and Patrimonial Relationships

\section{Agathe Baroin}

\section{OpenEdition}

Journals

Édition électronique

URL : https://journals.openedition.org/medievales/7109

DOI : 10.4000/medievales.7109

ISSN : $1777-5892$

Éditeur

Presses universitaires de Vincennes

Édition imprimée

Date de publication : 1 décembre 2013

Pagination : 93-107

ISBN : 978-2-84292-396-9

ISSN : 0751-2708

\section{Référence électronique}

Agathe Baroin, "Le couple en droit au haut Moyen Âge : autour de l'affectio maritalis et des relations patrimoniales », Médiévales [En ligne], 65 | automne 2013, mis en ligne le 20 janvier 2014, consulté le 22 avril 2022. URL : http://journals.openedition.org/medievales/7109 ; DOI : https://doi.org/10.4000/ medievales.7109 
Médiévales 65, automne 2013, p. 93-108

Agathe BAROIN

\section{LE COUPLE EN DROIT AU HAUT MOYEN ÂGE: AUTOUR DE L'AFFECTIO MARITALIS ET DES RELATIONS PATRIMONIALES}

À l'heure où la société, les juristes et le législateur se penchent sur les mutations de la famille et sur la définition du couple, il paraît opportun d'envisager le couple dans sa dimension historique afin d'essayer de répondre à une question essentielle: qu'est-ce que le couple ? ${ }^{1}$

Gérard Cornu, dans son Vocabulaire juridique, donne une définition du couple intéressante pour les historiens: "Le couple est l'union que forment un homme et une femme entre lesquels existent des relations charnelles (copula carnalis) et, en général, une communauté de vie, soit en mariage (couple légitime, union conjugale), soit hors mariage, en concubinage ou dans les liens d'un PACS (sous la précision que, depuis la loi du 15 novembre 1999, les partenaires de ces deux sortes d'unions peuvent être du même sexe) ${ }^{2}$.» Le couple ainsi déterminé dépasse donc largement le mariage tel qu'il a été défini par la doctrine chrétienne classique, définition reprise par les codificateurs du XIX ${ }^{\mathrm{e}}$ siècle dans un but d'organisation sociale et de «gouvernement des familles». En fait, il apparaît que les problématiques soulevées par la définition du couple contemporain rappellent les incertitudes liées à la détermination du couple altimédiéval. À cet égard, la période franque est riche d'enseignements: héritière de traditions juridiques romaines et chrétiennes, mais fortement influencée par des coutumes encore très vivaces dans le domaine du droit privé, la société franque constitue un excellent laboratoire pour l'étude du couple.

En effet, lorsque l'on étudie les sources normatives mérovingiennes, on s'aperçoit que les Francs saliens n'ont pas défini, ni même encadré le mariage;

1. Je remercie E. SAntinelli-FoltZ et S. Joye de leurs remarques.

2. G. Cornu, Vocabulaire juridique, Paris, 2011. 
toute la difficulté réside donc dans le fait que, sur le territoire de la Gaule mérovingienne, le mariage n'est pas institutionnalisé et qu'il ne le sera pas vraiment avant la période carolingienne. Il en va autrement dans les royaumes voisins, où les lois consacrent des titres entiers aux questions matrimoniales.

La comparaison des sources normatives et narratives avec la norme religieuse énoncée dans les canons des conciles va permettre d'expliquer comment l'Église a progressivement investi le champ matrimonial laissé vacant par le pouvoir royal, jusqu'à encadrer entièrement les relations de couple à partir de l'époque carolingienne. En effet, le pouvoir spirituel va s'engouffrer dans le vide juridique laissé par le pouvoir temporel en comprenant tout l'intérêt qu'il y a à encadrer les unions, de manière à mettre fin aux pratiques trop éloignées de ses propres règles en matière de parenté.

Sur une période allant de la fin du $v^{e}$ siècle jusqu'au $x^{e}$ siècle, nous retiendrons deux thèmes qui permettront de montrer les spécificités du couple altimédiéval du point de vue du droit: les relations personnelles et les rapports patrimoniaux du couple.

\section{Le couple, une communauté de vie?}

Les sources du premier Moyen Âge utilisent, entre autres expressions, le terme copula ou le verbe copulare pour désigner l'entrée dans le lien conjugal, qui pouvait se matérialiser par des cérémonies réglées par la coutume et réunissant les parentés des conjoints. Pour comprendre les relations matrimoniales qui soustendent le couple au haut Moyen Âge, nous allons successivement envisager le couple dans le mariage et le couple confronté à l'adultère.

\section{Le couple dans le mariage}

Dans le formulaire de Marculf, probablement compilé à la fin du $\mathrm{VII}^{\mathrm{e}}$ siècle ${ }^{3}$, deux formules concernant une donation mutuelle entre conjoints et un divorce présentent la communauté conjugale comme un synonyme de la communauté constituée par le couple (consortium coniugali et consortium (copule $)^{4}$.

L'utilisation du terme copula par les sources montre la difficulté de dissocier couple et mariage au premier Moyen Âge, probablement parce que

3. A. JEAnNIn, Formules et formulaires. Marculf et les praticiens du droit au premier Moyen $\hat{A} g e\left(V^{e}-X^{e}\right.$ s.), thèse, Lyon, 2007 ; A. Rio, The Formularies of Angers and Marculf. Two Merovingian Legal Handbooks, Liverpool, 2008, p. 107-113.

4. Marculfi formularum, I, 12 (éd. A. UdDholm, Marculfi formularum libri duo, Uppsala, 1962 , p. 64 et 272). 
les sources ne donnent pas de définition juridique de la formation du lien matrimonial; en outre, ni le couple ni le mariage ne sont institutionnalisés, ils ne constituent ni une institution étatique, ni une institution chrétienne, dans la mesure où la royauté et l'Église n'imposent aucune forme. Cette caractéristique n'est pas propre au haut Moyen Âge; à Rome, l'union matrimoniale se concluait par des cérémonies familiales, mais le droit ne les imposait pas à peine de nullité. En effet, les juristes romains ne concevaient pas le mariage comme une institution juridique, mais plutôt comme une situation de fait qui avait une portée sociale ${ }^{5}$. Dès lors, comment pouvait-on distinguer un mariage d'une liaison passagère? Pour qu'il y ait mariage, il suffisait qu'il y ait l'affectio maritalis, c'est-à-dire le consentement, la volonté des deux époux de vivre ensemble, durablement, et d'avoir des enfants. L'affectio maritalis marquait donc l'intention des parties de vivre une relation stable, durable, laquelle faisait toute la différence entre le mariage et l'union passagère ${ }^{6}$.

L'expression complète ne se retrouve guère dans les sources des $\mathrm{VI}^{\mathrm{e}}$ et $\mathrm{VII}^{\mathrm{e}}$ siècles; tout au plus trouvons-nous la mention, dans le Liber iudiciorum wisigothique, d'un homme qui ne veut pas dénoncer ni se séparer de sa femme adultère, parce qu'il ne souhaite pas rompre la communauté et l'affection conjugales, «coniugis consortio vel dilectione ${ }^{7} »$. En revanche, cette idée d'une union consentie, stable, «communauté de toute une vie», propre au justum matrimonium, se retrouve $\mathrm{au} \mathrm{IX}^{\mathrm{e}}$ siècle, tout particulièrement dans l'œuvre d'Hincmar de Reims, qui est le premier à élaborer une véritable doctrine du mariage. Sur le fond, Hincmar reprend certaines caractéristiques du mariage romain, telles que la stabilité, l'égalité de condition sociale, la liberté, mais il y ajoute la publicité - reprenant à son compte les dispositions du concile de Ver (755) - et la consommation de l'union, dessinant ainsi les contours d'un legitimum coniugium chrétien qui se distingue de plus en plus des autres types d'unions conjugales ${ }^{8}$.

5. P. Pichonnaz, Les Fondements romains du droit privé, Paris/Zurich, 2008, p. 152; J. Gaudemet, Le Mariage en Occident, Paris, 1987, p. 30.

6. A. BISCARDI, «Mariage d'amour et mariage sans amour en Grèce, à Rome et dans les Évangiles», Mélanges A. Biscardi, Scritti di diritto greco, Milan, 1999, p. 243 sq.; J. GAUdemet, «Union libre et mariage dans la Rome impériale», dans Le Droit de la famille en Europe. Son évolution depuis l'Antiquité jusqu'à nos jours. Actes des Journées internationales d'histoire du droit (Strasbourg, 23-26 mai 1991), Strasbourg, 1992, p. 375-392.

7. Liber iudiciorum III, 4, 13 (éd. K. ZeUMER, MGH, Leges nationum Germanicarum, I: Leges Visigothorum, Hanovre, 1902, p. 152-155).

8. Lettre d'Hincmar de Reims aux évêques d'Aquitaine (éd. E. Perels, MGH, Epistolae Karolini aevi, VI, 1, Hanovre, 1939, nº 136, plus particulièrement p. 92-93 et 96); J. Devisse, Hincmar, archevêque de Reims (845-882), t. I, Genève, 1975-1976, p. 396-408. 
Cependant, au $\mathrm{VI}^{\mathrm{e}}$ siècle, au sein même de la royauté, les prescriptions canoniques ne sont guère appliquées: les rois pratiquent la polygynie et contractent des unions dites incestueuses, considérées par les clercs comme autant d'infractions commises par les rois à l'encontre des canons. Ces pratiques ne sont pas aléatoires, les rois suivant en cela un ordre matrimonial qui apparaît au travers des noms donnés aux enfants royaux et qui assure leur prééminence sur les Francs. Il n'en reste pas moins que plusieurs de ces unions ont souvent été considérées par les clercs comme de simples concubinages.

L'exemple le plus flagrant est certainement celui de la première union de Clovis : de sa première épouse, mère de Thierry ${ }^{\text {er }}$, nous ne savons presque rien, mais Grégoire de Tours la qualifie, de manière très laconique, de concubine ${ }^{9}$. Pourtant, le nom donné à son fils, Thierry, reliait ce dernier à la fois à la lignée de sa mère et de son père, ce qui laisse supposer que l'origine de la première épouse de Clovis était illustre ${ }^{10}$; en outre, la répartition de l'autorité royale à la mort de Clovis a laissé à Thierry un territoire extrêmement étendu. Ainsi, en 511, Thierry reçut l'autorité sur les régions correspondant à l'ancien royaume de Cologne - anciennes provinces romaines de Germanie Première, Germanie Seconde et Belgique Première -, qui l'acceptèrent d'autant mieux comme roi que sa mère, princesse franque rhénane, lui avait transmis une légitimité royale. Des territoires conquis par son père, il acquit les principales villes de Champagne, l'Auvergne, ainsi que les villes d'Aquitaine qu'il avait soumises pour son père, c'est-à-dire Limoges, Cahors, Albi, Rodez, Mende et Javols. De par son étendue, le royaume de Thierry dépassait largement les royaumes de ses frères qui semblaient exercer leur pouvoir sur trois régions dont la superficie était équivalente. N'en déplaise à Grégoire de Tours, qui nie le rôle joué par la mère de Thierry dans l'étendue du pouvoir dont son fils hérita, c'est entre les trois fils de Clotilde que l'on tint «la balance égale ${ }^{11}$ », Thierry ayant été mis à la tête d'un royaume presque deux

9. GréGoIRe de Tours, Decem libri historiarum, II, 28 (éd. B. Krusch, W. Levison, Gregorii episcopi Turonensis Libri historiarum X, MGH, Scriptores rerum merovingicarum I, Hanovre, 1965).

10. Le nom de Thierry/TiuOdRih renvoyait à des origines illustres et à l'onomastique royale des Francs rhénans. Sur le lien entre les noms de personnes et la conscience familiale, K. F. WERNER, «Liens de parenté et noms de personne. Un problème historique et méthodologique», dans G. DuBY, J. Le Goff éd., Famille et parenté dans l'Occident médiéval. Actes du colloque de Paris (6-8 juin 1974), Rome/Paris, 1977, p. 13-18 et 25-34; H. Le BouRdELLES, «L'anthroponymie dans la famille de Clovis », dans M. Rouche éd., Clovis histoire et mémoire. I. Le baptême de Clovis, l'événement, Paris, 1997, p. 805-816; R. LE JAN, «Dénomination, parenté et pouvoir dans la société du haut Moyen Âge ( $\mathrm{vi}^{\mathrm{e}}-\mathrm{X}^{\mathrm{e}} \mathrm{s}$.) », dans EAD., Femmes, pouvoir et société dans le haut Moyen Âge, Paris, 2001, p. $224-$ 238 ; J.-P. Poly, Le Chemin des amours barbares, Paris, 2003, p. 258-271. Sur l'origine de la mère de Thierry, E. EwIG, «Die Namengebung bei den ältesten Frankenkönigen und im merowingischen Königshaus », Francia, 18/1 (1991), p. 21-69 (p. 49-50).

11. GrÉGOIRE DE Tours, Decem libri historiarum, III, 1 (éd. B. KrusCh, p. 97; trad. R. Latouche, Histoire des Francs, t. I, Paris, rééd. 1999, p. 142). 
fois plus grand que celui de ses demi-frères. Les modalités de la filiation royale montrent que les règles de validité de l'union que l'évêque de Tours aurait souhaité voir imposées n'étaient pas acceptées par la royauté: les deux unions de Clovis attestent de l'existence de mariages multiples, non hiérarchisés et ne correspondant pas aux critères matrimoniaux chrétiens ou même romains ${ }^{12}$. Enfin, le statut social de la première épouse de Clovis, le patrimoine et le pouvoir reçus par le fils de cette dernière, incitent à douter du statut de concubine d'une femme avec laquelle Clovis avait choisi de s'unir en premier. Si Grégoire de Tours a utilisé la notion romaine de concubina pour désigner la mère de Thierry, c'est surtout pour mettre en valeur Clotilde, épouse chrétienne qui avait amené Clovis au baptême, et pour faire de ce dernier le premier roi franc catholique et monogame.

Cet exemple permet de comprendre pourquoi l'affectio maritalis, élément essentiel du justum matrimonium, ne se retrouve pas dans les sources mérovingiennes. Le «bon mariage», le mariage «légitime» de la tradition romano-chrétienne ne correspondait pas aux réalités mérovingiennes. Pour autant, les couples mérovingiens pouvaient constituer des unions stables, dans lesquelles la femme avait toute sa place, éventuellement renforcée par ses origines. Cette situation particulière de la femme mérovingienne au sein de son couple apparaît encore plus nettement lorsque l'on considère le sort réservé aux femmes adultères.

\section{Le couple face à l'adultère}

Dans cette perspective, c'est l'adultère de la femme qui va plus spécialement nous intéresser. À Rome, comme dans toute l'Antiquité, un devoir de fidélité s'impose aux deux conjoints, mais seul l'adultère féminin est juridiquement sanctionné; en fait, le droit ne connaît pas l'adultère de l'homme. Au premier Moyen Âge, le Liber iudiciorum wisigoth consacre un titre entier aux adultères ${ }^{13}$; le texte n'envisage que l'adultère de la femme, mais l'homme qui a eu des relations sexuelles avec une femme mariée est lui aussi poursuivi. Certaines de ces lois prévoient que la femme et son amant soient livrés au mari qui pourra en

12. Sur les notions de Muntehe et Friedelehe qui ont encore été récemment reprises par des historiens contemporains, voir l'étude d'A. Esmyol, Geliebte oder Ehefrau? Konkubinen im frühen Mittelalter, Cologne, 2002, qui remet justement en cause ces termes que l'on ne retrouve pas dans les textes et qui constituent une construction historiographique; cependant, l'auteur ne s'interroge pas sur l'utilisation qui est faite du terme concubina par les auteurs mérovingiens. R. M. KARRAS, «The History of Marriage and the Myth of Friedelehe», Early Medieval Europe, 14/2 (2006), p. 119-151, revient sur le terme de Friedelehe et affirme qu'il n'existe pas de «distinction juridique formelle en matière matrimoniale» à l'époque mérovingienne.

13. Liber iudiciorum III, 4: «De adulteriis », p. 146-158. La date de rédaction du Liber iudiciorum a suscité de nombreuses études; en dernier lieu, C. MARTIN, «Le Liber iudiciorum et ses différentes versions», Mélanges de la Casa de Velázquez, 41/2 (2011), p. 17-34. 
disposer librement; la cause est ainsi entendue dans le cadre domestique et le meurtre de la femme adultère et de son complice est autorisé et n'est donc pas considéré comme un crime. Le Liber constitutionum burgonde consacre plusieurs articles à l'adultère féminin. La sévérité de la loi à l'égard des adultères est, là encore, évidente: il est ainsi prévu que l'homme bafoué pourrait tuer sa femme adultère et l'amant de celle-ci. Si l'amant acceptait de prêter serment qu'il n'était pas au courant de l'engagement antérieur de la femme, il ne subissait aucune peine: seul le délit commis en connaissance de cause était réprimé ${ }^{14}$.

Tout autre était le traitement réservé à l'adultère par les Francs. Si l'on considère le Pactus legis salicae, on constate que le terme d'adulterium n'est pas utilisé dans le sens «d'union extraconjugale», mais désigne un acte considéré comme relevant de la luxure. De même, les canons des conciles mérovingiens qualifient d'adulterium tout type de relations sexuelles considérées comme déviantes ${ }^{15}$. Il faut ajouter que les capitulaires des rois mérovingiens n'abordent pas la question de l'adultère. Enfin, les principaux auteurs de l'époque mérovingienne utilisent le terme d'adulterium, mais essentiellement pour exprimer la débauche; les femmes ne sont jamais vraiment condamnées pour avoir commis l'adultère ${ }^{16}$.

Le silence des sources normatives et narratives signifie peut-être que l'adultère féminin n'était pas puni publiquement, ne relevait pas d'une répression officielle, mais qu'il était sanctionné à l'intérieur de la domus, dans le cadre du couple conjugal. Cependant, une autre hypothèse est possible si l'on considère les accusations d'adultère proférées à l'encontre des reines mérovingiennes.

À la fin $\mathrm{du} \mathrm{vl}^{\mathrm{e}}$ siècle, deux cas d'infidélité réginale sont rapportés par les sources et ils incriminent à chaque fois la reine Frédégonde ${ }^{17}$. Ainsi, peu avant 580, Leudaste, comte de Tours, accusa Grégoire, évêque de Tours, de calomnier Frédégonde en prétendant qu'elle entretenait des relations adultères avec Bertrand, évêque de Bordeaux. Chilpéric, mari de Frédégonde, réunit à

14. Liber constitutionum, 52 (éd. L. R. von SALIS, MGH, Leges nationum Germanicarum, II, 1 : Leges Burgundionum, Hanovre, 1992, p. 85-87).

15. Tours II (567), 15 (éd. J. GaUdemet, B. Basdevant, Les Canons des conciles mérovingiens (VI $I^{e}-V I I^{e}$ s.), t. II, Paris, 1989, p. 360-361).

16. Grégoire de Tours rapporte le cas d'une femme adultère dont l'amant a tué le mari; ni l'un ni l'autre ne sont inquiétés. En revanche, à chaque fois que l'évêque de Tours relate des affaires dans lesquelles sont impliqués un clerc et une femme, que celle-ci soit mariée ou non, la femme est mise à mort, soit par sa famille, soit par son mari (GRÉGOIRE DE TouRs, Decem libri historiarum, VI, 13, VI, 36 et VIII, 19, éd. B. Krusch, p. 283, 307 et 385-386). N. PANCER, Sans peur et sans vergogne. De l'honneur et des femmes aux premiers temps mérovingiens, Paris, 2001, p. 198-207.

17. Les critiques de Grégoire de Tours à l'égard de Chilpéric et Frédégonde sont bien connues: I. N. Wood, «The Secret Histories of Gregory of Tours», Revue belge de philologie et d'histoire, 71/2 (1993), p. 253-270 ; D. Shanzer, «History, Romance, Love, and Sex in Gregory of Tours' Decem libri historiarum », dans K. Mitchell, I. N. Wood éd., The World of Gregory of Tours, Leyde, 2002, p. 395-418. 
Berny un concile rassemblant les évêques de son royaume afin de juger l'évêque de Tours. Celui-ci fut reconnu innocent des crimes qu'on lui imputait et c'est le calomniateur, Leudaste, qui fut frappé d'excommunication pour avoir porté une fausse accusation. Une seconde affaire éclata peu de temps après la mort de Chilpéric, en 584: Frédégonde fut accusée d'avoir enfanté le futur Clotaire II avec un leude. C'est le roi Gontran, frère de Chilpéric, très certainement poussé par les grands de son royaume, qui profère l'accusation et refuse de recevoir Clotaire II pour son baptême, «à moins de découvrir à son sujet des indices certains». Accusée, Frédégonde fut soutenue par les principaux grands de son royaume. En effet, trois cents grands et trois évêques se joignirent à elle en tant que cojureurs: ils affirmèrent tous par serment que l'enfant avait été engendré par Chilpéric et «ainsi le soupçon fut enlevé de l'esprit du roi », conclut Grégoire de Tours ${ }^{18}$.

Le dénouement de ces affaires d'adultère ne laisse pas d'étonner: dans le premier cas, Chilpéric, toujours en vie, prend la défense de sa femme avec ardeur et celle-ci n'est pas inquiétée, c'est son calomniateur qui est excommunié par l'assemblée des évêques. Le second cas est peut-être encore plus étonnant: le serment purgatoire prêté par la reine pour se disculper apparaît presque comme une manifestation d'autorité réginale au regard du nombre de fidéjusseurs venus se porter garants de la sincérité de l'accusée. Il faut souligner qu'habituellement, dans le cadre d'un serment purgatoire, les cojureurs ne déposent pas sur les faits de la cause; or, dans cette affaire, ils se prononcent sur la paternité de l'enfant.

Des enjeux politiques, ayant pour but de déstabiliser la royauté, sous-tendent ces accusations; cependant, l'attitude singulière de la société mérovingienne à l'égard de l'adultère féminin peut aussi éclairer un des aspects de la parenté chez les premiers Francs. Alors que tous les peuples qui les entourent condamnent l'infidélité féminine parce qu'elle risque d'introduire un sang étranger dans la famille, les Francs ne semblent pas y attacher une grande importance. Cette apparente liberté sexuelle laissée à la femme dans le cadre du couple conjugal pourrait être le signe du maintien d'un système de parenté cognatique, dans lequel la filiation maternelle a autant d'importance que la filiation paternelle.

À partir du $\mathrm{VIII}^{\mathrm{e}}$ siècle, l'adultère est davantage pris en compte par les textes ${ }^{19}$. L'adultère de la femme constitue pour les autorités ecclésiastiques

18. GréGoIre de Tours, Decem libri historiarum V, 47-49 et VIII, 9 (éd. B. Krusch, p. 257 262 et 376 ; trad. R. LATOUCHE, t. I, p. 314-322 et t. II, p. 136).

19. Les accusations d'adultère à l'encontre des reines se multiplient: au début du VIII ${ }^{\mathrm{e}}$ s., l'auteur du Liber historiae Francorum, non content de clouer au pilori Frédégonde et Faileuba, rapporta que Childéric avait commis l'adultère avec Basine, la femme du roi des Thuringiens. Cette accusation jetait l'opprobre sur toute la dynastie mérovingienne, probablement dans le but d'exalter la noblesse et de légitimer les Pippinides, nouveaux maîtres de la Francie (Liber historiae Francorum, 7, éd. B. Krusch, MGH, Scriptores rerum merovingicarum, II, Hanovre, 1888, p. 249). Sur l'adultère 
- qui se fondent en cela sur l'incise de Matthieu - un motif de renvoi de la femme par son mari ${ }^{20}$. Au IX ${ }^{\mathrm{e}}$ siècle, les textes ecclésiastiques interdisent le remariage de l'épouse et de l'époux séparés pour cause d'adultère de la femme; en outre, des pénitences sont désormais imposées aux fautifs, très certainement pour éviter les séparations et pour maintenir l'unité du couple chrétien défini par l'Église ${ }^{21}$. Le pouvoir temporel édicte des normes qui contribuent, elles aussi, à sanctionner l'adultère de la femme et de l'homme ${ }^{22}$. Cependant, cette égalité des deux sexes face à la répression n'est qu'apparente; en témoigne le capitulaire de Worms de 829, qui prévoit qu'un mari qui aura tué sa femme sans qu'elle ait commis d'adultère devra effectuer une pénitence ${ }^{23}$. Le texte laisse entendre que le meurtre de la femme coupable ne requiert aucune sanction, ce qui implique que l'adultère de l'épouse relève de la répression familiale ou, plus certainement, de celle du mari, dans le cadre même du couple conjugal, malgré les tentatives d'encadrement de ces pratiques par les autorités ecclésiastiques. À la fin du $\mathrm{IX}^{\mathrm{e}}$ siècle, la compétence ecclésiastique en matière d'adultère se renforce, l'Église exerce un contrôle plus grand sur les unions matrimoniales et redéfinit les contours de la famille autour du couple conjugal.

Au regard de la vie matrimoniale, la femme occupe, au sein du couple mérovingien, une place qui n'est pas celle des autres femmes des royaumes voisins ni celle de la femme carolingienne. L'analyse des rapports patrimoniaux au sein du couple confirme la pleine capacité de la femme au haut Moyen Âge.

\section{Le couple, une communauté d'intérêts?}

Au moment où se forme le couple, des biens sont échangés entre les familles de l'homme et de la femme ou directement entre ces derniers. Les transferts patrimoniaux mentionnés dans les sources sont nombreux : en dehors des présents échangés avant l'union, on trouve un don initial d'un sou et un denier fait à l'épouse et à ses parents par le futur mari, un morgenga $b$ versé par le mari à sa femme, puis les deux prestations principales que sont la dot versée

réginal à l'époque carolingienne, G. BÜHRER-THIERRY, «La reine adultère», Cahiers de civilisation médiévale, 35 (1992), p. 299-312.

20. Synode de Soissons (744), 9 (Pippini principis capitulare suessionense, éd. A. Boretius, MGH, Leges, Capitularia regum Francorum, I, Hanovre, 1883, nº 12, p. 30).

21. Second statut épiscopal de Théodulf d'Orléans, V, 5 et 6 (éd. P. Brommer, MGH, Capitula episcoporum, I, Hanovre, 1984, p. 162-163): il est prévu une pénitence de sept ans, dont trois ans au pain et à l'eau pour la femme et pour le mari adultères.

22. Hlotharii, Hludowici et Karoli conventus apud Marsnam secundus (851), 5 (éd. A. Boretius, V. Krause, MGH, Leges, Capitularia regum Francorum, II, Hanovre, 1897, nº 205, p. 73); repris dans le Conventus de Confluentes (860), 5, ibid., n 242, p. 155.

23. Capitulare pro lege habendum Wormatiense (829), 3 (ibid., nº 193, p. 18-19). 
par le mari à la future épouse et celle versée par le père de cette dernière à sa fille. Tous ces biens ne semblent pas versés systématiquement ${ }^{24}$, mais il est parfois difficile de savoir lesquels relèvent de tel ou tel type de transfert patrimonial. Au patrimoine reçu au moment de la formation de l'union s'ajoutent des biens acquis pendant la vie de couple: des donations peuvent être faites entre mari et femme, par des tiers et l'on rencontre une tertia, gain de survie de la femme. L'évolution de la nature et de l'étendue des droits de la femme sur ces biens au cours de la période franque permet d'appréhender le fonctionnement du couple conjugal quant à la gestion de son patrimoine et conduit à envisager la capacité juridique de l'épouse.

\section{Une pleine propriété de la femme sur ses biens à l'époque mérovingienne}

Ce sont les actes de la pratique qui nous renseignent le mieux sur la question. En effet, on trouve dans le Formulaire de Marculf une lettre de don nuptial, un libellus dotis, qui fait état des biens donnés par le père du marié à sa future belle-fille. La liste des biens meubles et immeubles transférés à la femme est éloquente: une villa, avec une maison d'habitation et tous les privilèges attachés à ladite villa, d'autres propriétés rurales, des esclaves, de l'or, de l'argent et du bétail. Tous ces biens sont donnés à la jeune femme en pleine propriété : ils sont sous sa domination et il lui appartient d'en faire ce qu'elle souhaite ${ }^{25}$. Le formulaire d'Angers comporte également une lettre de donation nuptiale de l'homme à sa future épouse, mais le transfert de propriété ne concerne qu'une $\operatorname{casa}^{26}$.

Dans certaines formules, la dos est cédée en usufruit: c'est le cas lorsque le mari n'est pas propriétaire des biens dont il cède l'usufruit à sa femme (par

24. La loi ripuaire (milieu du $\mathrm{VII}^{\mathrm{e}} \mathrm{s}$.) 41,2 , prévoit les cas où une dos ex marito n'a pas été constituée par un acte écrit au moment du mariage; il est alors prévu que la femme qui survit à son mari doit recevoir une somme de 50 solidi, un tiers des biens acquis par les époux durant le mariage et la totalité des biens qui lui ont été versés au titre de son morgengab. Ce texte pose la question de la dos ex marito comme condition de validité et preuve du mariage (Lex Ribuaria, éd. F. BEYERLE, R. BuCHNER, MGH, Leges nationum Germanicarum, III, 2 : Lex Ribuaria, Hanovre, 1954, p. 95). Sur le caractère obligatoire de la dos au moment de la conclusion du mariage: J. GAUDEMET, Le Mariage en Occident, p. 104-105; R. LE JAN, «Douaires et pouvoirs des reines en Francie et en Germanie (vi ${ }^{-}-\mathrm{X}^{\mathrm{e}} \mathrm{s}$.)», dans F. Bougard, L. Feller et R. Le JAN éd., Dots et douaires dans le haut Moyen Âge, Rome, 2002, p. 457-497; EAD., Famille et pouvoir dans le monde franc (VII ${ }^{e} \mathrm{X}^{e}$ s.). Essai d'anthropologie sociale, Paris, 1995, p. 351-352; E. SANTINelli, Des femmes éplorées? Les veuves dans la société aristocratique du haut Moyen Âge, Villeneuve-d'Ascq, 2003, p. 75-76.

25. Marculfi formularum II, 15 (éd. A. UddHoLm, p. 85).

26. Angers, 34 (Formulae Andecavenses, éd. K. Zeumer, MGH, Leges, Formulae Merowingici et Karolini aevi, Hanovre, 1886 [1963], p. 16). 
exemple, lorsqu'il est installé en tant que colon sur une terre ${ }^{27}$. On justifie parfois l'usufruit par la volonté d'avoir des enfants qui devront hériter des biens donnés par leur père à leur mère à la mort de cette dernière; cette disposition vise à empêcher l'appauvrissement du patrimoine familial, mais a également pour conséquence de limiter la capacité patrimoniale de la femme dans le couple ${ }^{28}$.

En étudiant les transferts patrimoniaux entre époux dans les formulaires $\mathrm{du} \mathrm{vi}^{\mathrm{e}}$ au $\mathrm{x}^{\mathrm{e}}$ siècle, Josiane Barbier a montré qu'il y avait autant de formules de libelli dotis faisant état d'une donation en usufruit qu'en pleine propriété et elle ne voit pas d'évolution chronologique sur la période. Cependant, elle précise qu'outre des spécificités géographiques - la majeure partie des dotations en pleine propriété se trouvant dans les régions de droit salique -, il existe des spécificités sociales. Ainsi, la pleine propriété est plus volontiers conférée par les grands, les dotations les plus importantes étant données en pleine propriété ${ }^{29}$. Faut-il alors voir dans la pleine propriété une pratique des grands qui, disposant de biens suffisants, pouvaient se permettre d'en céder une partie à leur épouse? Cette tendance pourrait être confirmée par des sources diplomatiques et narratives qui font état de biens donnés à des reines en toute propriété: villa de Saix donnée à Radegonde par Chilpéric; domaines laissés par Dagobert à Nanthilde, parmi lesquels la villa de Lagny; monastère de Chelles construit sur les biens propres de Bathilde; villae de Morsain et Cuisy données à Brunehaut par Childebert; enfin, cités données à Galswinthe par Chilpéric ${ }^{30}$. Cette dernière donation appelle

27. Angers, 40, p. 17-18.

28. Le Liber constitutionum burgonde, dans son titre 24 (éd. L. R. von SALIS, p. 61-62), prévoit que la veuve qui s'est remariée n'aura qu'un droit d'usufruit sur sa donatio nuptialis, sa dotation revenant, à sa mort, aux enfants de son premier époux (\$1). Si le premier époux n'avait pas d'enfant, la dotation devait revenir pour moitié à la famille de l'épouse et pour moitié à la famille de l'époux (§ 2). À la fin du vi ${ }^{\mathrm{e}} \mathrm{s}$., un édit de Chilpéric $\mathrm{I}^{\mathrm{er}}$ comporte des dispositions similaires puisqu'il prévoit que, si un homme sans enfant meurt, sa femme doit recevoir la moitié de sa dos, l'autre moitié allant aux parents de l'époux décédé; si la femme meurt et qu'il n'y a pas d'enfant, le mari reçoit la moitié de la dos et l'autre moitié va aux parents de la femme (Chilperici edictum [561-584], 5, éd. A. Boretius, MGH, Leges, Capitularia regum Francorum, I, Capitularia merowingica, $\mathrm{n}^{\circ} 4$, Hanovre, 1883, p. 8).

29. J. BARBIER, «Dotes, donations après rapt et donations mutuelles. Les transferts patrimoniaux entre époux dans le royaume franc d'après les formules ( $\mathrm{VI}^{\mathrm{e}}-\mathrm{XI}^{\mathrm{e}} \mathrm{s}$.) », dans F. BougarD et al. éd., Dots et douaires..., p. 353-388 (p. 363-365).

30. Baudonivie, Vita sanctae Radegundis, 3 (éd. B. KRusch, MGH, Scriptores rerum merovingicarum, II, Hanovre, 1888, p. 380). La villa de Saix est restée la propriété du monastère Sainte-Croix de Poitiers jusqu'à la Révolution; Gesta Dagoberti regis (éd. B. KRusch, MGH, Scriptores rerum merovingicarum, II, p. 423); Vita Balthildis A, 7 (éd. B. Krusch, MGH, Scriptores rerum merovingicarum, II, p. 489); Acte de Brunehaut pour Saint-Médard de Soissons entre 575 et 613 et second acte par lequel, à la demande de l'évêque de Soissons, Ansericus, Théodebert II confirme à la basilique Saint-Médard le legs de la villa de Morsain par son aïeule Brunehaut, entre 600 et 612 (éd. J. BARBIER, «Les actes royaux mérovingiens pour Saint-Médard de Soissons: une 
quelques remarques. En effet, les Dix livres d'histoires donnent un aperçu des nombreux biens donnés par le roi Chilpéric à Galswinthe au moment de leur mariage. Selon Grégoire de Tours, Galswinthe - fille du roi wisigoth Athanagild et sœur de Brunehaut - aurait alors acquis, au titre de sa dos et de son morgengab, les cités de Bordeaux, Limoges, Cahors, Benarnum et Begorra, avec tous leurs territoires et leur population. À la mort de Galswinthe sans enfant, en 568, les cités ne sont pas repassées sous la domination de Chilpéric mais furent attribuées, du vivant du roi, à la sœur de la défunte, la reine Brunehaut. Cet arrangement ne satisfit pas les parties. Un pacte fut alors passé à Andelot en 587 - trois ans après la mort de Chilpéric - entre Gontran, Brunehaut et Childebert II. Par ce pacte, il fut décidé que Brunehaut recevrait en pleine propriété la cité de Cahors avec tous ses territoires et sa population, alors que Gontran recevrait les autres cités en usufruit sa vie durant; à la mort de ce dernier, lesdites cités devraient repasser, dans leur totalité, sous la domination de Brunehaut et de ses héritiers ${ }^{31}$. Le pacte d'Andelot se présente ainsi comme la reconnaissance et l'affirmation des droits réels de Brunehaut sur les propriétés de sa sœur morte sans descendance.

L'importance des biens composant la dos de Galswinthe peut s'expliquer si l'on considère que ces biens portaient à la fois sur des choses corporelles et incorporelles; cependant, une autre explication peut être avancée. En effet, les biens détenus par Galswinthe pourraient être rapprochés de la catégorie romaine des res nec mancipi, dans la mesure où ils sont situés en dehors du territoire des premières conquêtes mérovingiennes. Ainsi, la localisation des biens reçus par Galswinthe permettrait de comprendre comment la reine a pu acquérir et transmettre à sa propre famille la maîtrise d'un territoire aussi étendu: toutes les cités étaient localisées en Aquitaine, région nouvellement conquise, voisine du royaume des Wisigoths dont était originaire Galswinthe et qui, en outre, avait été constamment morcelée et n'avait pas connu d'unité politique sous l'autorité d'un unique roi ${ }^{32}$. Toutes proportions gardées, un parallèle peut être établi entre la dotation d'une reine comme Galswinthe et les biens immeubles et meubles conséquents donnés en toute propriété aux femmes de l'aristocratie franque dont témoignent les formules. Enfin, nous avons la trace de biens donnés en toute propriété par le roi.

révision. Suivi d'un catalogue des actes royaux et privés pour Saint-Médard de Soissons antérieurs à 751 », dans D. Defente éd., Saint-Médard. Trésors d'une abbaye royale, Paris, 1997, actes 5 et 6 du catalogue).

31. Grégoire de Tours, Decem libri historiarum, IX, 20 (éd. B. Krusch, p. 437; trad. R. Latouche, t. II, p. 209-210). Sur le contexte historique du mariage de Galswinthe et du Pacte d'Andelot, voir B. Dumézil, La Reine Brunehaut, Paris, 2008, p. 160-162 et 240-247.

32. Sur la localisation de ces biens par rapport à l'espace wisigothique: C. MARTin, La Géographie du pouvoir dans l'Espagne visigothique, Villeneuve-d'Ascq, 2003, p. 290. 
L'étendue de leurs biens conférait à ces femmes une certaine indépendance patrimoniale au sein du couple, mais en contrepartie de cette indépendance, la femme richement dotée devait peut-être aussi s'attendre à une moindre protection de la part de son mari. Les textes montrent aussi que les femmes mérovingiennes pouvaient avoir la propriété de leurs biens dont elles disposaient librement; il semblerait même que, jusqu'au $\mathrm{IX}^{\mathrm{e}}$ siècle, la pleine propriété de la femme sur ses biens était la règle chez les grands et au sein de la famille royale. Le peu d'informations concernant ces biens ne nous permet pas de savoir avec certitude qui les administrait: leur gestion se faisait-elle en couple, par le mari seul ou par la femme seule? Un épisode rapporté par Grégoire de Tours laisse entendre que les femmes disposaient librement de leurs biens: au moment du mariage de sa fille, Frédégonde lui fit don de «grands trésors» provenant de biens qu'elle avait fait fructifier par son travail et dont son mari semblait ignorer l'existence ${ }^{33}$.

À partir de l'époque carolingienne, la marge de manœuvre des femmes au sein de leur couple tend à se réduire, avec la généralisation de la concession de la dotation en usufruit.

\section{La généralisation de l'usufruit à l'époque carolingienne}

Certes, à l'époque carolingienne, des biens sont encore donnés à la femme en pleine propriété. C'est le cas des reines mais aussi des femmes issues de familles de grands qui peuvent avoir une propriété entière sur leur $d_{o s}{ }^{34}$. Cependant, les biens donnés à la femme par son mari sont de plus en plus souvent concédés en usufruit ${ }^{35}$; la pratique s'est généralisée à tous les niveaux de la société, y compris au sein de la royauté. Dès le milieu du IX ${ }^{\mathrm{e}}$ siècle, on trouve un certain nombre d'actes royaux qui font état de donations d'un roi à sa femme en

33. Grégolre de Tours, Decem libri historiarum, VI, 45, éd. B. Krusch, p. 318.

34. Acte de Charles III le Simple en date du 19 avril 907, par lequel il concède à sa femme Frérone, à titre de dos, deux fiscs, Corbeny et Ponthion en Perthois, pour qu'elle en dispose comme bon lui semble (éd. P. LAuER, Recueil des actes de Charles III le Simple, roi de France (893-923), Paris, 1949, n 56, p. 120-123). Les formules de Bourges, d'époque carolingienne, et celles de Sens rédigées entre 768 et 774 contiennent des dispositions similaires tant par la nature des biens donnés que parce qu'ils sont transférés en pleine propriété à la future épouse par le mari: Bourges, 15a (Formulae Bituricenses, éd. K. Zeumer, p. 174-175); Sens, 25 (Formulae Senonenses, éd. K. Zeumer, p. 196). R. LE JAN, «Douaires et pouvoirs des reines en Francie et en Germanie ( $\mathrm{VI}^{\mathrm{e}}-\mathrm{X}^{\mathrm{e}}$ siècle)», p. 463.

35. C. Lauranson-Rosaz, «Douaire et sponsalicium durant le haut Moyen Âge», dans M. PARISSE éd., Veuves et veuvage dans le haut Moyen Âge, Paris, 1993, p. 102-103; R. LE JAN, «Aux origines du douaire médiéval ( $\mathrm{vi}^{\mathrm{e}}-\mathrm{x}^{\mathrm{e}}$ siècle)», M. PARISSE éd., La Veuve et le veuvage dans le haut Moyen Âge, Paris, 1993, p. 107-122. 
usufruit, sa vie durant ${ }^{36}$. Plus généralement, à partir du IX siècle, les donations de biens en usufruit à des reines - en dot ou à d'autres titres - se multiplient et, est-ce un hasard de la documentation, elles sont beaucoup plus nombreuses dans la partie orientale de l'ancien empire carolingien ${ }^{37}$.

À la mort de la reine, ces biens allaient, pour certains, à des monastères, mais beaucoup restaient dans la famille royale, revenant aux enfants communs du couple, à des parentes ou même au roi lui-même s'il survivait à sa femme. Désormais, l'usufruit est également pratiqué au sein de la famille royale, probablement dans le but de préserver le patrimoine royal ; on constate d'ailleurs que, dans certains cas, la dos ex marito a pour but de servir la politique royale en affirmant la présence des Carolingiens dans une région disputée ${ }^{38}$.

Plus généralement, la recherche d'une certaine stabilité du patrimoine familial est à mettre en relation avec l'encadrement des unions et avec l'emprise croissante de l'Église qui tente d'imposer l'indissolubilité du mariage. En effet, la stabilité des couples ne peut se faire sans une stabilité du patrimoine, lequel passe par une restriction des droits de propriété de la femme sur ses biens.

La progression de l'usufruit à l'époque carolingienne peut être rapprochée de la pratique de la tertia ${ }^{39}$. Les premières mentions d'un «tiers » apparaissent dans le Liber constitutionum burgonde, qui prévoit que la femme sans enfant, qui ne se remarie pas, pourra posséder à la mort de son mari le tiers des acquêts de ce dernier ${ }^{40}$; au $\mathrm{VII}^{\mathrm{e}}$ siècle, la loi ripuaire se préoccupe de la veuve, puisqu'elle oblige le mari qui n'aurait pas doté sa femme par acte solennel à lui assurer, à

36. Donation (16 mars 848) de Lothaire $\mathrm{I}^{\mathrm{er}}$ à sa femme Ermengarde du monastère Saint-Sauveur de Brescia (éd. T. SchIEFFER, MGH, Diplomata, Die Urkunden Lothars I. und Lothars II., Berlin, 1966, n 101); donation (10 février 878) de Charles III le Gros à sa femme Richarde des monastères de Seckinga et Saint-Félix et Regula, à Zurich, puis du monastère de Pavie et de l'abbaye de Zurzach en 881 (éd. P. KeHr, MGH, Diplomata, Die Urkunden Karls III., Berlin, 1937, nº 7, p. 42-43).

37. L'empereur Arnulf donne à une parente, Rotrude, en bénéfice, sa vie durant, le domaine de Brumath en Alsace (acte du 27 novembre 889, éd. P. KeHR, MGH, Diplomata, Die Urkunden Arnolfs, Berlin, 1940, nº 70, p. 105-106); le même donne à l'église de Salzbourg le domaine d'Ardienga, que sa mère, Liutswinde, avait détenu en bénéfice (acte du 9 mars 891, n 87, p. 128-130); Louis III le Jeune, fils de Louis II le Germanique, donne à l'abbaye de Lorsch le domaine d'Aberinesbourg, qui avait été détenu par son épouse Liutgarde (acte du 18 janvier 882, éd. P. KeHR, MGH, Diplomata, Die Urkunden Ludwigs des Deutschen, Karlmanns und Ludwigs des Jüngeren, Berlin, 1956, $\mathrm{n}^{\circ} 24$, p. 364-365); Louis IV l'Enfant donne des domaines que sa mère, Oda, avait reçus de son père, Arnulf; de même il donne à sa mère des domaines qui doivent, à la mort de cette dernière, revenir à ses sœurs (éd. T. SchiEFFer, MGH, Diplomata, Die Urkunden Zwentibolds und Ludwigs des Kindes, Berlin, 1963, n 28, p. 138-140 et $\mathrm{n}^{\circ} 52$, p. 177).

38. R. LE J JN, «Douaires et pouvoirs des reines...», p. 473.

39. Sur la tertia, E. SANTinelli, Des femmes éplorées? ..., p. 77-79.

40. Liber constitutionum, 42,1 , p. 73 (le tiers pourra aussi lui être assuré par son fils unique: Liber constitutionum, 62, 1 ; elle peut également revendiquer un tiers des propres de son mari : Liber constitutionum, 74, 1). 
titre de gain de survie, le tiers des conquêts ${ }^{41}$. Au début du IX $\mathrm{X}^{\mathrm{e}}$ siècle, l'auteur des Gesta Dagoberti interpole un passage de la Chronique de Frédégaire et attribue à la reine Nanthilde, épouse de Dagobert I ${ }^{\text {er }}$, un gain de survie constitué d'un tiers des acquêts de son mari ${ }^{42}$. Enfin, la tertia entre officiellement dans le système normatif franc en 821, dans un capitulaire de Louis le Pieux ${ }^{43}$. Le texte prévoit qu'à la mort de son mari, la veuve a droit au tiers des biens acquis en bénéfice durant leur vie commune, à titre de tertia pars conlaborationis.

La tertia constitue un véritable gain de survie octroyé à la veuve qui, plus que la dos ex marito, préfigure le douaire médiéval. En effet, la dos ex marito ne constitue pas uniquement un gain de survie; la femme devient propriétaire ou usufruitière de ses biens au moment de son mariage et elle peut les aliéner ou en percevoir les fruits pendant sa vie de couple. En revanche, la tertia paraît n'être qu'un gain de survie, dont la femme ne dispose qu'à la fin de sa vie, à la mort de son époux. La tertia, associée à la pratique de l'usufruit dotal, annonce ainsi le douaire ${ }^{44}$.

L'époque franque est marquée par une évolution très importante des structures de parenté qui se caractérise par une conjugalisation de la famille. De fait, la réalité du couple altimédiéval peut être appréhendée en abordant la question de la place de la femme dans le couple. En effet, la femme jouit d'une certaine indépendance: dans sa vie conjugale d'abord, où elle ne semble pas contrainte à une très stricte obligation de fidélité, puis dans ses rapports patrimoniaux avec son conjoint, qui lui offrent une grande liberté d'action. Cependant, dans la société du haut Moyen Âge, cette indépendance n'est pas sans conséquences: la liberté de mœurs suscite les accusations d'adultère et la libre disposition de biens conséquents rend moins nécessaire la protection traditionnelle du mari ou de la parentèle. Enfin, le resserrement des familles autour du couple conjugal, particulièrement à partir de l'époque carolingienne, rend nécessaire la protection matérielle de la veuve au moyen d'un gain de survie.

Agathe BARoIn - Université de Reims Champagne-Ardenne, CEJESCO (EA 4693, Reims), Département de Droit, 57 rue Pierre-Taittinger, 51096 Reims Cedex

41. Lex Ribuaria, 41, 2, p. 95.

42. Gesta Dagoberti, 46 (éd. B. Krusch, MGH, Scriptores rerum Merovingicarum, II, Hanovre, 1956, p. 422).

43. Capitula missorum (821), 9 (éd. A. Boretius, MGH, Leges, Capitularia regum Francorum, I, Hanovre, 1883, p. 301).

44. En ce qui concerne le mot «douaire», M. Parisse, «Conclusion», dans F. Bougard et al. éd., Dots et douaires..., p. 537, souligne les changements intervenus dans la situation des femmes et leur dotation à la fin du $\mathrm{x}^{\mathrm{e}} \mathrm{s}$. 


\section{Le couple en droit au haut Moyen Âge : autour de l'affectio maritalis et des relations patrimoniales}

Héritière de traditions juridiques romaines et chrétiennes, mais fortement influencée par des coutumes encore très vivaces dans le domaine du droit privé, la société franque constitue un excellent laboratoire pour l'étude du couple. La comparaison des sources normatives et narratives avec la norme religieuse énoncée dans les canons des conciles va permettre d'expliquer comment l'Église a progressivement investi le champ matrimonial laissé vacant par le pouvoir royal, jusqu'à encadrer entièrement les relations de couple à partir de l'époque carolingienne. Sur une période allant de la fin du v viècle jusqu'au $\mathrm{X}^{\mathrm{e}}$ siècle, l'article explore deux thèmes qui permettent de montrer les spécificités du couple altimédiéval du point de vue du droit: les relations personnelles et les rapports patrimoniaux du couple.

mariage - droit - affectio maritalis - patrimoine - douaire

\section{The Couple in Front of the Law in the Early Middle Ages : Affectio Maritalis and Patrimonial Relationships}

The Frankish society, which is heiress of both Roman and Christian legal traditions, but strongly influenced by customs in the field of private law, constitutes an excellent laboratory to study the couple. The comparison between the normative, narrative sources and the councils explains how the Church gradually got to gain control over the marriage. From the end of the fifth century to the tenth century, this article investigates two themes which show the specificities of the early medieval couple from a legal point of view: the personal and the patrimonial relationships between husband and wife.

marriage - law - affectio maritalis - patrimony - dowry 
